\title{
Sustained release of milrinone delivered via microparticles in a rodent model of myocardial infarction
}

\author{
Hamood Al Kindi, MD, ${ }^{a}$ Arghya Paul, PhD, ${ }^{b}$ Zhipeng You, PhD, ${ }^{\mathrm{c}}$ Oleg Nepotchatykh, PhD, ${ }^{\mathrm{d}}$ \\ Adel Schwertani, $\mathrm{PhD},{ }^{\mathrm{c}}$ Satya Prakash, $\mathrm{PhD},{ }^{\mathrm{e}}$ and Dominique Shum-Tim, MD ${ }^{\mathrm{a}}$
}

Objective: The aim of the present study was to construct a new drug delivery system for milrinone using microparticles. This novel technology enhances drug bioavailability and decreases toxicity, with future implications for the treatment of end-stage heart failure.

\begin{abstract}
Methods: Polylactic-co-glycolic acid microparticles (PLGA-MPs) loaded with milrinone were prepared using a double emulsion-solvent evaporation technique. In vitro release kinetics was evaluated at physiologic conditions. A total of 24 female Lewis rats underwent left coronary artery ligation. One week after ligation, all rats were randomized to 1 of 3 groups ( $n=8$ per group). Group I received an intravenous injection of PLGA-MPs alone; group II, a bolus intravenous injection of milrinone; and group III an intravenous injection of milrinone-PLGA-MPs. All injections were administrated slowly by way of the tail vein over 10 minutes. Transthoracic echocardiography, noninvasive heart rate monitoring, and blood pressure measurements were performed at different predetermined intervals before and for 24 hours after the injection. All rats survived for 24 hours and were then killed by euthanasia. Serum plasma was taken for cytokine assays and determination of milrinone levels using high-performance liquid chromatography.
\end{abstract}

Results: Group III had a significantly greater left ventricular ejection fraction at 90 minutes and 3, 6, and 12 hours after treatment compared with the other groups. The milrinone plasma level was significantly greater in group III than in the other groups (group I, $0 \mathrm{ng} / \mathrm{mL}$; group II, $1.7 \pm 2.4 \mathrm{ng} / \mathrm{mL}$; group III, $9.1 \pm$ $2.2 \mathrm{ng} / \mathrm{mL} ; P<.05)$. The intercellular adhesion molecule and cytokine-induced neutrophil chemoattractant-1 levels were significantly lower in group III than in the other 2 groups $(P<.05)$.

Conclusions: Drug encapsulation using microparticles can prolong the effects of milrinone. We propose a new strategy for future drug delivery in patients with end-stage heart failure. (J Thorac Cardiovasc Surg 2014;148:2316-24)

Nanomedicine is a new multidisciplinary field that has demonstrated promising potential in medical therapeutic and diagnostic applications. This evolving field includes micro- and nanoparticles (MNPs) whose structures exhibit distinct physical, chemical, and biologic properties owing to their small size. These features make them highly valuable in biomedical applications as biologic markers,

From the Division of Cardiothoracic Surgery and Surgical Research, ${ }^{\mathrm{a}}$ Royal Victoria Hospital, McGill University Health Center, Montreal, Quebec, Canada; Department of Chemical and Petroleum Engineering, ${ }^{b}$ University of Kansas, Lawrence, Kan; Division of Cardiology, ${ }^{\mathrm{c}}$ Montreal General Hospital, Montreal, Quebec, Canada; Department of Chemistry, ${ }^{\mathrm{d}}$ McGill University, Montreal, Quebec, Canada; and Biomedical Technology and Cell Therapy Research Laboratory, ${ }^{\mathrm{e}}$ Department of Biomedical Engineering, Faculty of Medicine, McGill University, Montreal, Quebec, Canada.

Disclosures: Authors have nothing to disclose with regard to commercial support.

Read at the 94th Annual Meeting of The American Association for Thoracic Surgery, Toronto, Ontario, Canada, April 26-30, 2014.

Received for publication April 21, 2014; revisions received June 21, 2014; accepted for publication July 6, 2014; available ahead of print Aug 29, 2014.

Address for reprints: Dominique Shum-Tim, MD, Division of Cardiothoracic Surgery and Surgical Research, Royal Victoria Hospital, McGill University Health Center, 687 Ave des Pins Ouest, Room S-8-73b, Montreal, QC H3A 1A1, Canada (E-mail: dshumtim@yahoo.ca).

$0022-5223 / \$ 36.00$

Copyright (c) 2014 by The American Association for Thoracic Surgery

http://dx.doi.org/10.1016/j.jtcvs.2014.07.033 contrast agents for biologic imaging, and drug delivery systems. ${ }^{1}$ Drug encapsulation using MNPs could have the advantages of increasing the drug circulation time and passing across biologic barriers to allow subsequent internalization and distribution within tissues of interest through passive and active targeting mechanisms. ${ }^{2}$

Milrinone is a type III phosphodiesterase inhibitor that has strong inotropic and vasodilator effects. It has little effect on the heart rate and oxygen consumption, offering an advantage over other cardiac inotropes. ${ }^{3}$ Milrinone has been commonly used to treat low cardiac output and high pulmonary arterial pressure. ${ }^{4}$ It also exhibits an anti-inflammatory effect, such that its role in the management of systemic inflammatory response syndrome after cardiopulmonary bypass $^{5}$ and/or sepsis has become more apparent. ${ }^{6,7}$ The half-life of milrinone in humans is approximately 2 hours; thus, it is usually given by way of a central line as a continuous infusion in a monitored setting. ${ }^{8}$ Intravenous milrinone has been reported to have been given as an outpatient therapy for patients with end-stage heart failure who required frequent repeat hospitalizations, were inotropic dependent, or were awaiting either heart transplantation or implantation of a ventricular assist device..$^{9,10}$ The logistics of outpatient, intravenous administration of a 


\section{Abbreviations and Acronyms \\ CINC-1 = cytokine-induced neutrophil chemoattractant-1 \\ DDQ $=2,3$ dichloro-5,6-dicyanobenzoquinone \\ HPLC $=$ high-performance liquid chromatography \\ ICAM-1 = intercellular adhesion molecule-1 \\ IL-10 = interleukin 10 \\ LVEF = left ventricular ejection fraction \\ LVFS = left ventricular fractional shortening \\ MNPs = micro- and nanoparticles \\ MPs = microparticles \\ PLGA = polylactic-co-glycolic acid \\ TNF- $\alpha=$ tumor necrosis factor $-\alpha$}

potent inotrope requires tremendous paramedical support and is costly and potentially hazardous. The oral form of milrinone is less effective with less predictable outcomes, such that it is rarely used clinically. ${ }^{1}$

The objective of the present study was to construct a new drug delivery system for milrinone using biodegradable microparticles (MPs) to enhance drug bioavailability with controlled pharmacokinetics and to decrease the cytotoxicity and dosage frequency. This could potentially offer a novel therapeutic strategy, similar to insulin administration for patients with diabetes, for the treatment of patients with end-stage heart failure. We hypothesized that intravenous injection of milrinone prepared in biodegradable MPs might prolong its inotropic effect by sustained a slow-release mechanism that enhances myocardial function in a rat model of ischemic cardiomyopathy. To the best of our knowledge, this is the first study that delivered cardiac inotropes using biodegradable MP technology.

\section{METHODS}

\section{In Vitro Studies}

MP preparation. Biodegradable polylactic-co-glycolic acid (PLGA)-MPs were loaded with milrinone using a double emulsionsolvent evaporation technique. In brief, milrinone solution $(2.5 \mathrm{mg})$ was added to PLGA $(50 \mathrm{mg})$ dissolved in $1 \mathrm{~mL}$ dichloromethane. The primary emulsion was generated by a high-speed homogenizer (PowerGen 125; Thermo Fisher Scientific, Waltham, Mass) for 2 minutes. Double emulsion was achieved by adding the emulsion to $10 \mathrm{~mL}$ of $0.5 \%$ (wt/vol) polyvinyl alcohol and homogenizing again for another 1 minute. To remove the organic solvent, the resultant water-in-oil-in-water emulsion was placed under magnetic stirring for 3 hours at room temperature. After 3 hours, the hardened PLGA-MPs were then washed 3 times with distilled water by centrifugation. The MPs were prepared in 3 different batches and frozen at $-20^{\circ} \mathrm{C}$ for future use.

\section{Morphology and Structure Characterization of Microspheres}

The morphology of the microspheres was studied using scanning electron microscopy (Hitachi S-4700 FE; Hitachi, Chiyoda, Japan). The size was confirmed using a particle sizer instrument. The diameter of the MPs was measured using the technique of electrophoretic laser Doppler anemometry and a Zeta Potential Analyzer (Brookhaven Instruments Corp, Holtsville, NY). The ZetaPlus Particle Sizer software, version 4.11 (Brookhaven Instruments Corp) was used to determine the size distribution of the MPs. The particle sizes were measured for 3 batches of MPs, and each measurement was obtained after taking the average of the 3 runs.

\section{Milrinone Encapsulation Efficiency and Release Kinetics}

The percentage of encapsulated milrinone drug was estimated using the 2,3 dichloro-5,6-dicyanobenzoquinone (DDQ) test as described previously. ${ }^{12}$ In brief, DDQ was used as a oxidative coupling and charge transfer reagent to quantify the milrinone concentration using a spectrophotometer. The drug loading efficiency was determined by dissolving accurately weighed amounts of drug-carrying MPs (approximately $15 \mathrm{mg}$ ) in $5 \mathrm{~mL}$ acetonitrile and subsequent quantification by adding the DDQ solution $(0.05 \% \mathrm{wt} / \mathrm{vol})$ in methanol and reading the absorbance at $356 \mathrm{~nm}$. To detect the release kinetics, the drug-loaded MPs were placed in $10 \mathrm{~mL}$ phosphate-buffered saline and into glass vials. The containers were horizontally shaken at $37^{\circ} \mathrm{C}$. At predetermined intervals, $1-\mathrm{mL}$ samples were withdrawn (replaced with fresh phosphate-buffered saline) and treated with DDQ for quantification using spectrophotometry. Each experiment was conducted in triplicate.

\section{In Vivo Studies}

All experiments were performed on female Lewis rats (weight, 200-250 g; Charles River Laboratories, Wilmington, Mass) in accordance with the guidelines set forth by the Canadian Council on Animal Care and were approved by the institutional ethics committee.

\section{Ligation of Left Anterior Descending Coronary Artery and Intravenous MP Injection}

Ligation of the left anterior descending artery was performed through a left thoracotomy, as previously described. ${ }^{13}$ One week after ligation, all 24 rats were randomized into 1 of 3 groups. Group I received intravenous tail vein injection of empty PLGA-MPs $(250 \mu \mathrm{L})$. Group II received a bolus intravenous tail vein injection of milrinone $(50 \mu \mathrm{g} / \mathrm{kg}$ in $250 \mu \mathrm{L})$ slowly over 10 minutes. ${ }^{14,15}$ Finally, group III received an intravenous tail vein injection of milrinone prepared in PLGA-MPs in an equivalent dose and volume as in group II. The reason for using empty MPs as a control was to ensure that these particles per se had no effect on cardiac function or other inadvertent toxicity. Various endpoint measurements were taken as described in subsequent paragraphs. At 24 hours after tail vein injection, all rats were killed by euthanasia. The hearts were washed with phosphate-buffered saline solution to remove excess blood and clots and then fixed in neutral-buffered $4 \%$ formalin. Blood was collected and centrifuged at $1200 \mathrm{rpm}$ for 20 minutes, and the serum plasma was stored at $-80^{\circ} \mathrm{C}$ for additional analysis.

\section{Echocardiography, Heart Rate, and Blood Pressure Measurements}

Echocardiographic examinations and heart rate and blood pressure measurements were performed with the rats under inhaled isoflurane anesthesia ( $2.5 \%$ in oxygen, $500-700 \mathrm{~mL} / \mathrm{min}$ ). Baseline transthoracic echocardiography was performed on day 4 after left anterior descending artery ligation for each rat, followed by serial measurements, after tail vein injection, at the following intervals: 30 and 90 minutes, and 3, 6, 12, and 24 hours. Serial echocardiograms were performed in a blinded fashion using a commercially available system (Micromaxx P04224; SonoSite, Bothell, Wash), equipped with a linear probe, 7 to $13-\mathrm{MHz}, 25-\mathrm{mm}$ footprint, turbo transducer (P06519.11; SonoSite). The left ventricular end-diastolic and end-systolic 
diameters were measured from M-mode tracings between the anterior and posterior walls from the parasternal short-axis view just below the level of the papillary muscles. The time of end diastole was defined as the time of the maximum diameter of the left ventricle in 1 heart cycle. Accordingly, end systole was defined as the minimum diameter. The left ventricular fractional shortening (LVFS) and left ventricular ejection fraction (LVEF) were estimated using the American Society of Echocardiography leading-edge method, as previously described. ${ }^{16}$

The mean arterial blood pressure and heart rate measurements were obtained using the CODA standard noninvasive blood pressure system (Kent Scientific Corp, Torrington, Conn). The measurements were done serially after intravenous tail injection at 5, 15,30, and 90 minutes.

\section{Cytokine Measurement}

To determine the pro-inflammatory/anti-inflammatory cytokine ratio, we measured the plasma level of tumor necrosis factor (TNF)- $\alpha$ and interleukin (IL)-10 using enzyme-linked immunosorbent assay kits (Biosource, Invitrogen, Life Technologies, Norwalk, Conn) according to the manufacturer's recommendations. In addition, the relative cytokine level was detected in rat plasma using the Proteome Profiler Rat Cytokine Array Panel A (R\&D Systems, Minneapolis, Minn), as instructed by the manufacturer. The average signal (pixel density) of the pair of duplicate spots representing each cytokine was determined and subtracted from the background according to the manufacturer's recommendations. ${ }^{17}$

\section{Measurement of Milrinone Level Using High- Performance Liquid Chromatography}

Materials. Milrinone lactate, a $1-\mathrm{mg} / \mathrm{mL}$ solution for injection, was prepared (Sandoz Canada Inc, Boucherville, Quebec, Canada). Amrinone was used as an internal standard and was obtained from Sigma-Aldrich (Oakville, Ontario, Canada). High-performance liquid chromatography (HPLC)-grade methanol, HPLC-grade acetonitrile, phosphoric acid, and dipotassium phosphate were obtained from Fisher Scientific Inc (Ottawa, Ontario, Canada). Water was purified on site by reverse osmosis and Milli-Q (Millipore Corp, Billerica, Mass).

Chromatography. The HPLC system included the Beckman System Gold 126 binary pump, Beckman System Gold 166 variable wavelength detector, Beckman System Gold 508 autosampler and Flatron TC-50 column thermostat. The chromatographic data were collected and processed using 32 Karat software (Beckman Coulter Canada Inc, Mississauga, Ontario, Canada). The separation of milrinone lactate and amrinone was achieved using the Symmetry C18, $5 \mu \mathrm{m}, 4.7 \times 150 \mathrm{~mm}$, column (Waters, Milford, Mass). The mobile phase composition was $37.5 \%$ methanol and $62.5 \% 5 \mathrm{mM} \mathrm{K}_{2} \mathrm{HPO}_{4}$ buffer in Milli-Q water and was adjusted to $\mathrm{pH}$ 7.4 with $\mathrm{H}_{3} \mathrm{PO}_{4}$. The mobile phase was filtered on a Teflon $0.45-\mu \mathrm{m}$ filter and degassed before the use. It was used as an isocratic elution with the flow rate of $1.4 \mathrm{~mL} / \mathrm{min}$. Internal standard solution was prepared by dissolving $1.0 \mathrm{mg}$ of amrinone in $500 \mathrm{~mL}$ HPLC-grade acetonitrile. The amrinone solution had 2 functions - to introduce the internal standard into the sample and to precipitate plasma proteins. The standard solution of milrinone lactate was prepared by dilution of $1 \mathrm{mg} / \mathrm{mL}$ original milrinone lactate solution in Milli-Q water. A $200 \mu \mathrm{L}$ plasma aliquot was transferred into a $1.5-\mathrm{mL}$ polypropylene microcentrifuge tube and $800 \mu \mathrm{L}$ of amrinone solution was added before the tube was closed, vortexed at high speed for 1 minute, and centrifuged at $12,000 \mathrm{~g}$ for 3 minutes. After centrifugation, the supernatant was transferred into a clean glass test tube $(10 \times 75$ $\mathrm{mm}$ ) and evaporated at $60^{\circ} \mathrm{C}$ under a gentle flow of dry nitrogen. After complete liquid evaporation, $100 \mu \mathrm{L}$ of the mobile phase was added to the test tube and vortexed for 30 seconds. Next, the liquid was transferred into an HPLC vial with 100 to $150 \mu \mathrm{L}$ for analysis. The injection volume was $27 \mu \mathrm{L}$. Standard samples were prepared exactly the same way, with the only difference that, instead of plasma, the standard solution of milrinone lactate with a concentration of 1 to $1000 \mathrm{ng} / \mathrm{mL}$ was used.

\section{Method Validation}

The recovery of milrinone was estimated as $84 \%$, acceptable for the internal standard method. The detection limit was $2.5 \mathrm{ng} / \mathrm{mL}$ and the quantitation limit was $5 \mathrm{ng} / \mathrm{mL}$. The method is linear in the wide concentration range $(5-1000 \mathrm{ng} / \mathrm{mL})$, with the least squares weighting $1 /$ response 2 . The goodness of fit $\left(\mathrm{r}^{\wedge} 2\right)$ was 0.997 .

\section{Statistical Analysis}

All statistical analyses were performed using the Statistical Package for Social Sciences program, version 16 (SPSS, Chicago, Ill). All statistical tests were 2 -tailed. All data are presented as the mean \pm standard deviation. Repeated echocardiographic variables, heart rate, and blood pressure measurements were compared using 1-way repeated measures analysis of variance. In addition, analysis of variance was used to compare the cytokine and milrinone level among the groups at 24 hours after injection. If a significant F-ratio were obtained, a Bonferroni post hoc test was used to assess the pair-wise differences.

\section{RESULTS}

\section{Morphology and Structure Characterization of Microspheres}

Electron microscopic analysis confirmed the presence of MPs and provided morphologic information on typical milrinone-loaded PLGA-MPs (Figure 1, A). The particles were about 4 to $5 \mu \mathrm{m}$ in diameter. They had an oval shape and smooth surfaces. Loading the PLGA-MPs with milrinone did not result in any significant change in the shape or size of the MPs.

\section{Milrinone Encapsulation Efficiency and Release Kinetics}

The encapsulation efficiency of milrinone with different PLGA concentrations is shown in Figure 1, $B$. At a milrinone/PLGA concentration ratio of 1:10 and 1:20, the encapsulation efficiency was $16.45 \% \pm 4.3 \%$ and $23.62 \% \pm$ $3.3 \%$, respectively. No significant change was found in the size compared with the free MPs. Increasing the milrinone/PLGA concentration ratio to 1:40 resulted in improvement in the encapsulation efficiency to $27.96 \% \pm 5.6 \%$, with an increase in the MP size to $7.38 \pm 5.6$.

The percentage of milrinone release from the PLGA particles within 24 hours is illustrated in Figure 1, C. An initial burst effect release phase occurred, followed by a lag release phase, characteristic of PLGA-MPs. ${ }^{18,19}$ Changing the milrinone/PLGA ratio from 1:10 to $1: 40$ decreased the amount of milrinone released during the burst and lag phases. A milrinone/PLGA ratio of 1:20 was selected for the in vivo experiments because the encapsulation of milrinone at this concentration resulted in minor changes in the MP size and shape, with gradual and slow release of milrinone over 24 hours.

\section{Echocardiography, Heart Rate, and Blood Pressure Measurements}

Figure 2, $A$ and $B$, show the mean \pm standard deviation of the LVEF and percentage of LVFS measured for all the 


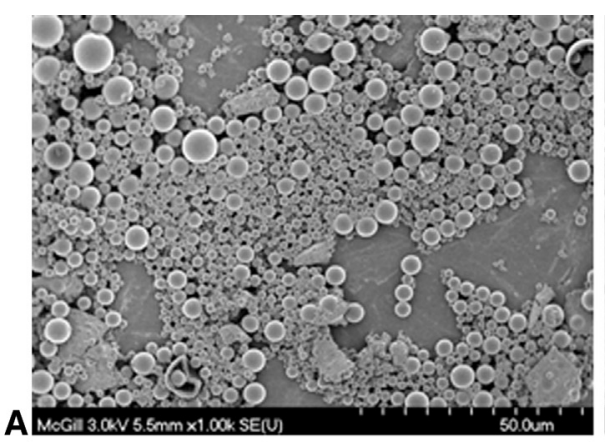

Free MP

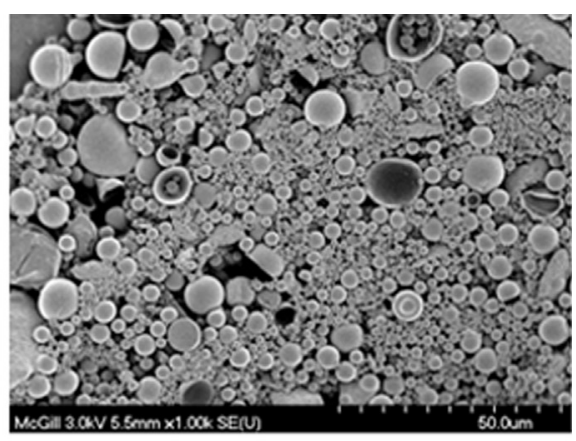

MP+Mil

\begin{tabular}{|c|c|c|}
\hline Milrinone: PLGA & Homogenization & Diameter \\
\hline $\mathrm{w} t / \mathrm{wt}$ & \%Encapsulation & $\mu \mathrm{m}$ \\
\hline $1: 10$ & $16.45 \pm 4.3$ & $4.20 \pm 1.5$ \\
\hline $1: 20$ & $23.62 \pm 3.3$ & $4.81 \pm 2.3$ \\
\hline $1: 40$ & $27.96 \pm 5.6$ & $7.38 \pm 5.6$ \\
\hline
\end{tabular}

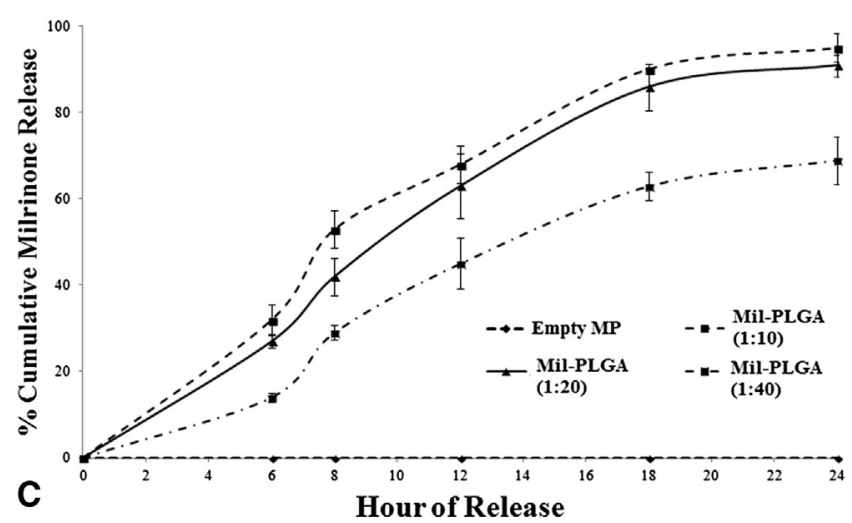

FIGURE 1. Characterization of microparticles. A, Transmission electron microscopy was used to obtain the size characterization. The polylactic-coglycolic acid $(P L G A)$ microparticles $(M P s)$ measured $\approx 4 \mu \mathrm{m}$ in diameter. Most microparticles were spherical in shape. B, Encapsulation efficiency using various milrinone (Mil)-PLGA concentrations. C, In vitro release kinetics of milrinone (Mil)-loaded PLGA microparticles over time. Mil-PLGA concentration of 1:20 was used in this study.

groups at the predetermined intervals. The LVEF and LVFS at baseline and after left anterior descending artery ligation were not significantly different among the groups $(P>.05)$. At 30 minutes, the mean LVEF and LVFS measured in group III (LVEF, 73\% $\pm 9 \%$; LVFS, $52 \% \pm 6 \%$ ) and group II (LVEF, $69 \% \pm 3 \%$; LVFS, $44 \% \pm 3 \%$ ) were significantly greater than those in group I (LVEF, $47 \% \pm$ $2 \%$; LVFS, $26 \% \pm 0.6 \% ; P<.05$ ). At 90 minutes, the mean LVEF and LVFS in group III (LVEF, $72 \% \pm 8 \%$; LVFS, $51 \% \pm 6 \%$ ) were significantly greater than those in both group I (LVEF, 46\% $\pm 3 \%$; LVFS, $25 \% \pm 1 \%$ ) and group II (LVEF, 60\% $\pm 7 \%$; LVFS, 36\% $\pm 5 \%$; $P<.05$ ). At 3, 6, and 12 hours, the mean LVEF and LVFS in group III had gradually decreased but were nevertheless significantly greater than in the other groups
$(P<.05)$. At 24 hours, group III had a tendency toward greater LVEF and LVFS recovery after injection compared with that in groups I and II, but the difference did not reach statistical significance $(P>.05)$. Figure $2, C$ and $D$, shows the mean and standard deviations of the mean arterial blood pressure and heart rate in all groups. One-way analysis of variance showed no significant differences among the groups at the predetermined intervals $(P>.05)$.

\section{Serum Cytokine Measurements}

The mean pro-inflammatory/anti-inflammatory cytokine ratio (TNF- $\alpha / \mathrm{IL}-10)$ measured in the plasma of all rats is shown in Figure $3, A$. The serum TNF- $\alpha /$ IL10 ratio was significantly greater in group I $(6.2 \pm 1.1)$ than in groups II and III $(4.2 \pm 0.4$ and $3.8 \pm 0.8$, respectively, $P<.05)$. 

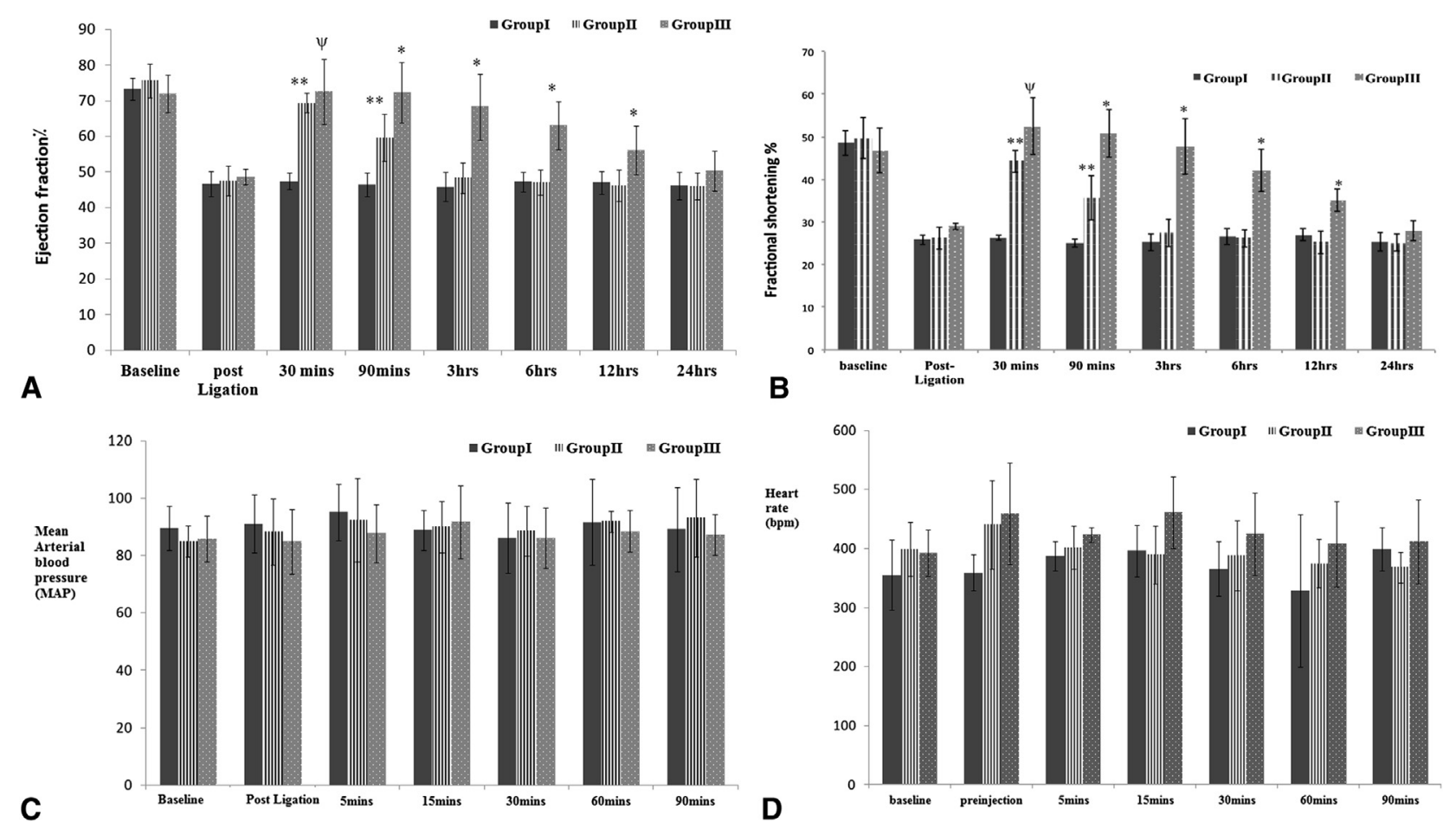

FIGURE 2. Hemodynamic measurements. Data presented as mean \pm standard deviation. A, Graphs of left ventricular ejection fraction determined by 2dimensional images. B, Graphs of percentage of fractional shortening determined by 2-dimensional images. C, Graphs of mean arterial pressure measurements. D, Graphs of heart rate measurements. ${ }^{*} P<.05$, group III versus groups I and II; ${ }^{\psi} P<.05$, group III versus group I; ** $P<.05$ group II versus group I.

Group III had a lower TNF- $\alpha /$ IL-10 ratio than group II, but the difference did not reach statistical significance $(P>.05)$.

The relative multicytokine panel is shown in Figure 3, $B$. Vascular endothelial growth factor and macrophage inflammatory protein- $3 \alpha$ signals were absent in groups II and III. The relative pixel density of cytokine-induced neutrophil chemoattractant-1 (CINC-1) and intercellular adhesion molecule-1 (ICAM-1) in group III (CINC-1, $0.86 \pm 0.02$; ICAM-1, $0.18 \pm 0.01)$ were significantly lower than those in group I (CINC-1, $1.03 \pm 0.02$; ICAM-1, $1.01 \pm 0.04$ ) and group II (CINC-1, $0.98 \pm 0.04$; ICAM-1, $0.31 \pm$ $0.01 ; P<.05)$.

\section{Milrinone Assay Using HPLC}

Both milrinone lactate and amrinone were detected at a wavelength of $325 \mathrm{~nm}$. The retention time of milrinone was 6.15 minutes and of amrinone (internal standard) was 3.93 minutes. The total run time was 8 minutes. HPLC analysis (Figure 4) showed that the milrinone plasma level at 24 hours after treatment in group III was significantly greater than that in groups I and II (group I, $0 \mathrm{ng} / \mathrm{mL}$; group II, $1.7 \pm 2.4 \mathrm{ng} / \mathrm{mL}$; group III, $9.1 \pm 2.2 \mathrm{ng} / \mathrm{mL} ; P<.05$ ).

\section{DISCUSSION}

The technologies of MNPs have advanced tremendously during the past 2 decades for their potential applications in various healthcare fields. MNPs promote the innovative creation of drug delivery systems that increase drug bioavailability, decrease toxicity, and enhance drug targeting by passive or active mechanisms. ${ }^{20}$ The US Food and Drug Administration has approved several drug delivery systems using these particles. Most of these drugs were used in the field of oncology, such as polyethylene glycol-stabilized liposomal doxorubicin (Doxil, Evacet), protein-bound paclitaxel (Abraxane), and pemetrexid (Alimta) to deliver a highly toxic drug in a target-specific site with minimal side effects. ${ }^{21}$ In cardiovascular drug delivery, MNPs have been used in different settings that focused mainly on the treatment of in-stent restenosis. ${ }^{22,23}$ In the present study, we have demonstrated the feasibility of using MPs to prolong the inotropic effect of a single injection of milrinone by a slow drug release mechanism attributed to the semipermeable properties of the polymer. To our knowledge, this is the first report to use MPs to deliver cardioactive drugs for the treatment of heart failure.

The MPs used in the present study for milrinone encapsulation were prepared from PLGA polymer, one of the most widely used Food and Drug Administration-approved biodegradable polymers. This polymer has the following attractive features: biocompatibility, well-described formulations and methods of preparation adapted to various types of hydrophilic (eg, milrinone) or hydrophobic drug 

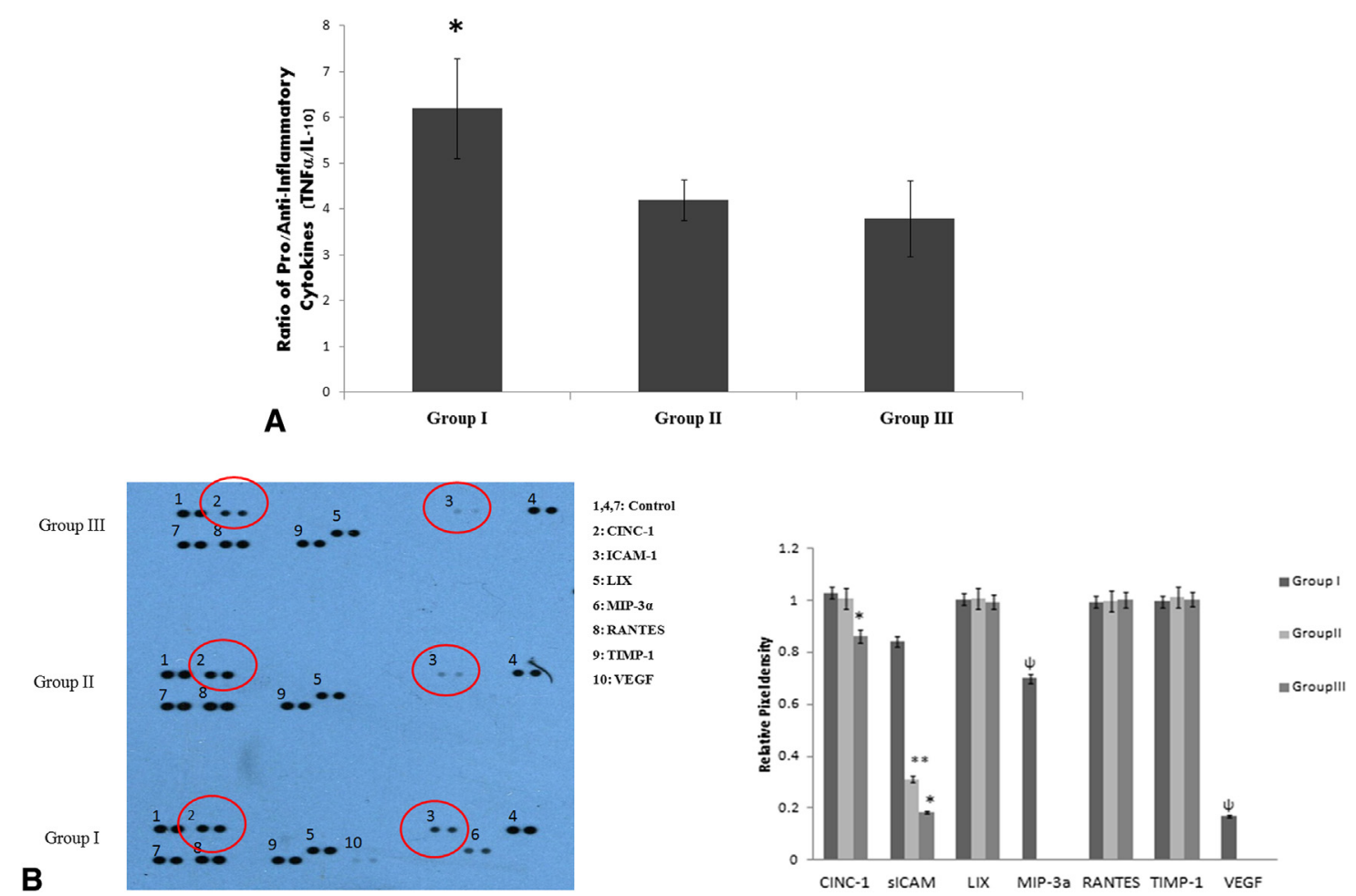

FIGURE 3. Plasma cytokine levels for group I (empty microparticles), group II (milrinone), and group III (microparticles plus milrinone). A, Ratio of serum tumor necrosis factor $(T N F)-\alpha$ to interleukin-10 levels. ${ }^{*} P<.05$, group I versus groups II and III. B, Rat cytokine panel. A, upper, Representative array images shown. Lower, Profiles, created by quantifying dot densities, background-subtracted, and normalized to positive controls, are presented. Note the signal intensities of serum intercellular adhesion molecule (ICAM) and cytokine-induced neutrophil chemoattractant-1 (CINC-1) in group III were significantly less than in the other groups. Macrophage inflammatory protein-3 $(M I P-3 \alpha)$ and vascular endothelial growth factor $(V E G F)$ were absent in groups II and III. $* P<.05$, group III versus groups I and II; $* * P<.05$, group II versus group I; ${ }^{\psi} P<.05$, group I versus groups II and III. $L I X$, Lipopolysaccharide-induced CXC chemokine; RANTES, chemokine ligand-5; TIMP-1, tissue inhibitors of metalloproteinase-1; IL-10, interleukin 10.

applications, the possibility for sustained release preparation, and protection of drugs from rapid degradation. ${ }^{24}$ The size of the PLGA-MPs used in the present study was about $4 \mu \mathrm{m}$, ideal for the intravenous route and providing sustained systemic release of the drug without the risk of embolization. Particles $>10 \mu \mathrm{m}$ will persist at the site of injection and would be appropriate for local delivery ${ }^{25}$; however, particles $<1 \mu \mathrm{m}$ will be ideal for targeted therapy because of their ability to cross the mucous barrier, endothelial cells, and even the blood-brain barrier. ${ }^{26}$ The release kinetics curve of milrinone in vitro showed an initial burst effect, characteristic of the PLGA MPs. The initial burst release is the quantity of the drug that escapes from the MPs before the onset of polymer erosion-mediated drug release. ${ }^{27}$ We found that the burst effect in vitro was at 6 to 7 hours; however, the increase in the LVEF after injecting milrinone-PLGA-MPs in the rat was early, within the first 30 minutes. This discrepancy could have been because milrinone is a hydrophilic drug, which will facilitate its diffusion to the surface of the particles, especially after entering the blood stream. ${ }^{28}$ The fast burst effect could be modified by wide particle size distributions or coating the
MPs with materials that can provide a protective layer to help in delaying the burst effect and increasing the circulation time. ${ }^{29}$ As such, many MNP-designed options are technically available to obtain the optimal pharmacokinetics desirable for drugs for different clinical purposes.

Milrinone has been widely used clinically in the treatment of low cardiac output, pulmonary hypertension, and right ventricular failure. It has a class IIb indication for continuous long-term support for patients with end-stage heart failure who are inotropic dependent and awaiting either transplantation or ventricular assist device implantation. ${ }^{30}$ In the present study, we have shown a significant recovery in left ventricular contractility in the group receiving milrinone-PLGA-MPs compared with the other groups for $\geq 12$ hours or, potentially, 24 hours. The effect was prolonged $\geq 4$ times longer than milrinone as a free drug given at the same dose and using the same route. No significant differences were found in the heart rate or mean arterial blood pressure among the groups. The milrinone dose used in the present study was arbitrarily similar to that used in the published data. Just as confirmed by Verrijk and colleagues ${ }^{31}$ at the same dosage used in our study, the 


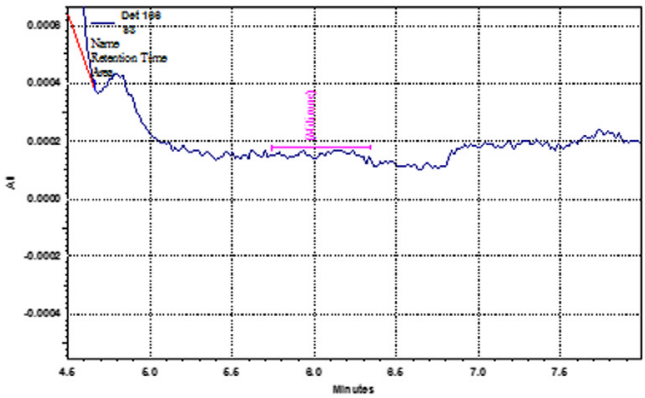

A

Group I

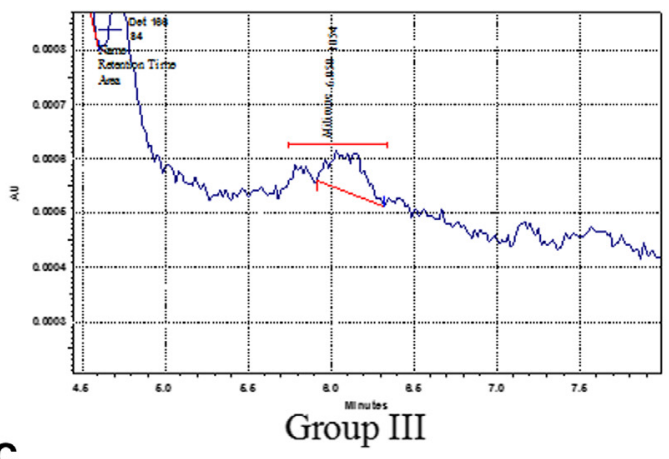

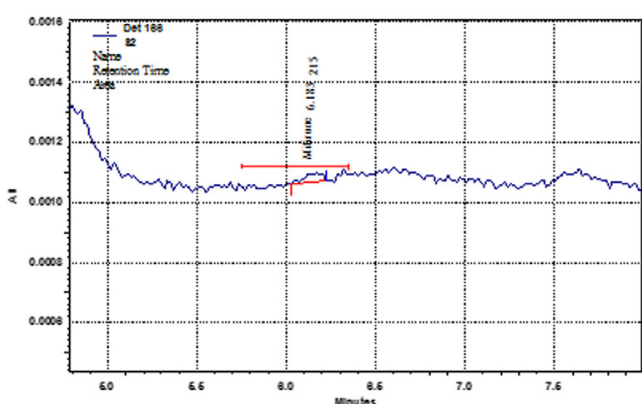

Group II
B

\begin{tabular}{|c|c|c|c|c|}
\hline Mean \pm SD & $\begin{array}{c}\text { Group I } \\
(\mathrm{n}=8)\end{array}$ & $\begin{array}{c}\text { Group II } \\
(\mathrm{n}=8)\end{array}$ & $\begin{array}{c}\text { Group III } \\
(\mathrm{n}=8)\end{array}$ & Pvalue \\
\hline $\begin{array}{c}\text { Mil level } \\
(\mathrm{ng} / \mathrm{ml})\end{array}$ & 0 & $1.7 \pm 2.4$ & $9.1 \pm 2.2$ & $\begin{array}{c}\text { P }<0.05 \\
\text { (Group III vs } \\
\text { Group I \& II) }\end{array}$ \\
\hline
\end{tabular}

D

FIGURE 4. High-performance liquid chromatography of milrinone in rat plasma. A, Group I (empty microparticles); B, group II (milrinone); and C, group III (microparticles plus milrinone). D, The mean plasma milrinone level in all 3 groups at 24 hours after tail vein injection. SD, Standard deviation.

change in blood pressure was minimal and the increase in the heart rate was mild. Apart from its strong inotropic effect, milrinone also has essential anti-inflammatory effects that have been reported in many experimental and clinical trials in the settings of myocardial infarction, congestive heart failure, cardiopulmonary bypass, and sepsis. These effects are probably indirectly related to the improvement in the hemodynamics after administering the drug. However, direct effects by elevating cyclic adenosine monophosphate and inhibiting the NF- $\kappa \mathrm{B}$ pathway, which will decrease the production of pro-inflammatory cytokines, have been suggested. $^{32,33}$ Similar to previous reports, we have shown that giving a single dose of milrinone, either as a free drug or prepared in MPs, could result in a significant reduction of the pro-inflammatory markers (TNF- $\alpha$, chemokines CINC-1, macrophage inflammatory protein-3 $\alpha$, ICAM, and vascular endothelial growth factor). In addition, encapsulation of milrinone using MPs prolonged its effect on the production of these cytokines, suggested by the significantly lower level of CINC-1 and ICAM in group III, despite group III having received the same dose of milrinone as group II.

The present study had several limitations. First, this was the first application of MNPs in the delivery of cardioactive drugs. The dose-response characteristics, optimal hemodynamic effects, and determination of unacceptable hemodynamic side effects need to be defined. Nevertheless, we have shown the feasibility of prolonging the inotropic effect of milrinone without increasing the toxicity or side effects. Second, no invasive hemodynamic monitoring was used to obtain objective data regarding cardiac output, blood pressure, heart rate, systemic resistance, pulmonary pressure, and so forth. Transthoracic echocardiography could potentially have created a bias, because it is operator dependent. To minimize this bias, the echocardiographic operator and analysis in the present study were blinded to the type of injection and treatment. Third, no detailed in vivo pharmacokinetics study was performed to document milrinone release over time. Finally, the variation in phosphodiesterase-3 expression across different species should also be considered. This might have accounted for the differences in inotropic effects on human versus rats. It has been reported that more inotropic effect will be seen at the same dose of milrinone in humans than in rats. ${ }^{34}$ This reiterates the need for additional studies to investigate the dose-response characteristics and species differences with milrinone.

\section{CONCLUSIONS}

The encapsulation of milrinone in MPs could provide new strategies for delivering these hemodynamically potent drugs in a more gentle and prolonged fashion and 
minimizing the hemodynamic side effects. Future studies are needed to optimize the encapsulation and milrinone release in a predictable manner. In addition, using PLGAMP technology could potentially develop different sustained-release milrinone formulations, such as an intramuscular, a subcutaneous, or an oral form that can be delivered by various routes. Inhaled milrinone-PLGA-MPs is one such example that sustained milrinone delivery can be achieved by nasal administration with minimal systemic side effects and toxicity. The application of MNPs in drug delivery has proved to be effective and feasible. Additional exploitation for cardiovascular application could hold great potential for the treatment of patients with congestive heart failure.

\section{References}

1. Xia Y. Nanomaterials at work in biomedical research. Nat Mater. 2008;7:758-60.

2. Devalapally H, Chakilam A, Amiji MM. Role of nanotechnology in pharmaceutical product development. J Pharm Sci. 2007;96:2547-65.

3. Young RA, Ward A. Milrinone. Drugs. 1988;36:158-92.

4. Hilleman DE, Forbes WP. Role of milrinone in the management of congestive heart failure. Ann Pharmacother. 1989;23:357-62.

5. Gong M, Lin X-Z, Lu G-T, Zheng L-J. Preoperative inhalation of milrinone attenuates inflammation in patients undergoing cardiac surgery with cardiopulmonary bypass. Med Princ Pract. 2011;21:30-5.

6. Rich N, West N, McMaster P, Alexander J. Milrinone in meningococcal sepsis. Pediatr Crit Care Med. 2003;4:394-5.

7. Barton P, Garcia J, Kouatli A, Kitchen L, Zorka A, Lindsay C, et al. Hemodynamic effects of IV milrinone lactate in pediatric patients with septic shock: a prospective, double-blinded, randomized, placebo-controlled, interventional study. Chest. 1996;109:1302-12.

8. Edelson J, Stroshane R, Benziger DP, Cody R, Benotti J, Hood WB Jr, et al. Pharmacokinetics of the bipyridines amrinone and milrinone. Circulation. 1986; 73(Pt 2):III145-52

9. Young JB, Moen EK. Outpatient parenteral inotropic therapy for advanced heart failure. J Heart Lung Transplant. 2000;19:S49-57.

10. Price JF, Towbin JA, Dreyer WJ, Moffett BS, Kertesz NJ, Clunie SK, et al. Outpatient continuous parenteral inotropic therapy as bridge to transplantation in children with advanced heart failure. $J$ Card Fail. 2006;12:139-43.

11. Packer M, Carver JR, Rodeheffer RJ, Ivanhoe RJ, DiBianco R, Zeldis SM, et al. Effect of oral milrinone on mortality in severe chronic heart failure. $N$ Engl $J$ Med. 1991;325:1468-75.

12. Siddiqui MR, Tariq A, Ahmad A, Chaudhary M, Shrivastava SM, Singh RK. Application of DDQ and p-chloranilic acid for the spectrophotometric estimation of milrinone in pharmaceutical formulations. Asian J Sci Res. 2009;2:135-45.

13. Al Kindi H, Adil H, Asenjo JF, Ge Y, Chen GY, Bhathena J, et al. Microencapsulation to reduce mechanical loss of microspheres: implications in myocardial cell therapy. Eur J Cardiothorac Surg. 2011;39:241-7.

14. Drexler H, Höing S, Faude F, Wollschläger H, Just H. Central and regional vascular hemodynamics following intravenous milrinone in the conscious rat: comparison with dobutamine. J Cardiovasc Pharmacol. 1987;9:563-9.

15. Huang M-H, Wu Y, Nguyen V, Rastogi S, McConnell BK, Wijaya C, et al. Heart protection by combination therapy with esmolol and milrinone at late-ischemia and early reperfusion. Cardiovasc Drugs Ther. 2011;25:223-32.

16. Davani S, Marandin A, Mersin N, Royer B, Kantelip B, Hervé P, et al. Mesenchymal progenitor cells differentiate into an endothelial phenotype, enhance vascular density, and improve heart function in a rat cellular cardiomyoplasty model. Circulation. 2003;108(Suppl 1):II-253.

17. Cady RJ, Denson JE, Durham PL. Inclusion of cocoa as a dietary supplement represses expression of inflammatory proteins in spinal trigeminal nucleus in response to chronic trigeminal nerve stimulation. Mol Nutr Food Res. 2013;57:996-1006.

18. Acharya G, Shin CS, Vedantham K, McDermott M, Rish T, Hansen K, et al. A study of drug release from homogeneous PLGA microstructures. J Control Release. 2010;146:201-6.

19. Allison SD. Analysis of initial burst in PLGA microparticles. Expert Opin Drug Deliv. 2008;5:615-28
20. Parveen S, Misra R, Sahoo SK. Nanoparticles: a boon to drug delivery, therapeutics, diagnostics and imaging. Nanomed Nanotechnol Biol Med. 2012;8: 147-66.

21. Faraji AH, Wipf P. Nanoparticles in cellular drug delivery. Bioorg Med Chem 2009; 17:2950-62.

22. Kolodgie FD, John M, Khurana C, Farb A, Wilson PS, Acampado E, et al. Sustained reduction of in-stent neointimal growth with the use of a novel systemic nanoparticle paclitaxel. Circulation. 2002;106:1195-8.

23. Margolis J, McDonald J, Heuser R, Klinke P, Waksman R, Virmani R, et al. Systemic nanoparticle paclitaxel (nab-paclitaxel) for in-stent restenosis I (SNAPIST-I) a first-in-human safety and dose-finding study. Clin Cardiol. 2007:30:165-70.

24. Danhier F, Ansorena E, Silva JM, Coco R, Le Breton A, Préat V. PLGA-based nanoparticles: an overview of biomedical applications. J Control Release. 2012;161:505-22.

25. Weiniger CF, Golovanevski L, Domb AJ, Ickowicz D. Extended release formulations for local anaesthetic agents. Anaesthesia. 2012;67:906-16.

26. Rytting E, Nguyen J, Wang X, Kissel T. Biodegradable polymeric nanocarriers for pulmonary drug delivery. Expert Opin Drug Deliv. 2008;5:629-39.

27. Jain RA. The manufacturing techniques of various drug loaded biodegradable poly(lactide-co-glycolide) (PLGA) devices. Biomaterials. 2000;21:2475-90.

28. Fredenberg S, Wahlgren M, Reslow M, Axelsson A. The mechanisms of drug release in poly (lactic-co-glycolic acid)-based drug delivery systems-a review. Int J Pharm. 2011;415:34-52.

29. Dinarvand R, Sepehri N, Manoochehri S, Rouhani H, Atyabi F. Polylactide-coglycolide nanoparticles for controlled delivery of anticancer agents. Int $J$ Nanomed. 2011;6:877.

30. Verrijk R, Vleeming W, Van Rooij HH, Wemer J, Porsius AJ. Plasma elimination of milrinone in rats in relation to its hemodynamic effects. J Pharm Sci. 1990;79: 236-9.

31. Goldhaber JI, Hamilton MA. Role of inotropic agents in the treatment of heart failure. Circulation. 2010;121:1655-60.

32. Barin JG, Čiháková D. Control of inflammatory heart disease by CD4+ T cells Ann N Y Acad Sci. 2013;1285:80-96.

33. Huang ZG, Jin Q, Fan M, Cong XL, Han SF, Gao H, et al. Myocardial remodeling in diabetic cardiomyopathy associated with cardiac mast cell activation. PloS One. 2013;8:e60827.

34. Weishaar RE, Kobylarz-Singer DC, Steffen RP, Kaplan HR. Subclasses of cyclic AMP-specific phosphodiesterase in left ventricular muscle and their involvement in regulating myocardial contractility. Circ Res. 1987;61:539-47.

\section{Discussion}

Dr Vivek Rao (Toronto, Ontario, Canada). Thank you. I have no conflicts to disclose.

I would like to congratulate you on a very clear presentation and a very interesting technological proof-of-concept study. You found that the encapsulation of milrinone in these MPs led to a sustained release of milrinone with potential beneficial effects.

I have 3 questions for you related to this study. The first is the PLGA that you used for the MPs appears to me to be lipophilic. In your in vitro release studies, you had phosphate-buffered saline. Have you done studies in which you actually have a mono layer of cells to determine whether the cells tend to accumulate this particle?

Dr Al Kindi. PLGA polymer is hydrophobic, but it can encapsulate the hydrophilic drugs by mechanical entrapment inside its highly porous meshlike structure. That is the idea of how this PLGA can release milrinone in a sustained way because it is lipophilic, so the release will be slowly over 24 hours.

Dr Rao. But can be it absorbed into a cell wall and have ....

Dr Al Kindi. Actually, the degradation of the PLGA in humans is by hydrolysis. So, the end result is lactic acid, and the lactic acid will be absorbed by the liver, and then that is how it is metabolized. That is why it is biodegradable and approved by the Food and Drug Administration. 
Dr Rao. My second question relates to the hemodynamic effects of both drug delivery systems. You mentioned that one of the limitations was a lack of invasive hemodynamic assessments. In the report, you documented that the heart rate and blood pressure did not differ among the 3 groups.

How do you reconcile that intravenous injection of milrinone did not cause tachycardia or a hypotensive effect, which is commonly seen clinically?

Dr Al Kindi. That is an excellent question. The way we measured the blood pressure and heart rate was a noninvasive method using a tail cuff. It is noninvasive method and can be affected by body temperature. We did not see any difference in the heart rate and blood pressure among all 3 groups. Probably, in future studies, we should measure the heart rate and blood pressure more precisely, using invasive methods.

Dr Rao. Finally, in your conclusion, as well as in your last slide, you tease us with the concept of using this alternative delivery technique. So, do you have any preliminary data that you can share with us in terms of prolonging the effect of milrinone therapy using subcutaneous, intramuscular, or inhalational therapy?

Dr Al Kindi. Actually, the size of the MPs we used in this project was $4 \mu \mathrm{m}$, which is ideal actually for the inhalation route.

That would also be a very good method to deliver milrinone and to decrease its systemic side effects, so that is why we are thinking along that line.

Dr David H. Harpole, Jr (Durham, NC). Excellent study, very interesting. I wanted to have you give us a little of your insight. We know that milrinone is truly an inotropic agent in the heart. It also, as you mentioned in the very beginning, has vasodilatory effects in the pulmonary circulation, it unloads the right heart. Actually, a number of investigators think that might be how it is more cardioprotective, because it actually unloads the heart.

Now, your microspheres were large enough that they probably would not be absorbed directly into the pulmonary circuit. You just mentioned this. If you changed your delivery system to inhaled with a slightly smaller particle, do you think you might also receive the benefit of the pulmonary unloading, which would help the right heart in failure?

Dr Al Kindi. It all depends on your treatment target. If your target is pulmonary hypertension, I think the inhalation method would be appropriate in this context.

If you are treating heart failure or you want to have the systemic effect of milrinone, perhaps the subcutaneous route or intramuscular route would be ideal. It all depends on your target.

Dr Todd K. Rosengart (Houston, Tex). It would appear that what you are showing us is that you can extend the duration of the effect from about 3 hours to 12 hours or so; however, perhaps what is more exciting about this, as you alluded, is that nanoparticles will allow the use of inhalation instead of the current formulation.

So, is this a change in the method of delivery or is the importance of your study the duration of effect, or is it something else?

Dr Al Kindi. This study is the first study to use MPs to deliver cardioactive drugs. So, we have to start with the intravenous route. However, ultimately we are working on delivering these MPs using a more convenient method. As I mentioned, we will start with the subcutaneous route and then to more difficult routes, such as the inhalation route, and that is our ultimate goal. 